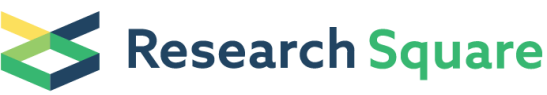 \\ Preprints are preliminary reports that have not undergone peer review. \\ They should not be considered conclusive, used to inform clinical practice, or referenced by the media as validated information.
}

\section{An acceptable methodological evaluation method of real-time fluorescent RT-PCR targeting SARS-CoV-2}

xiaodong ren ( $D$ 604456131@qq.com )

Third Military Medical University Daping Hospital and Research Institute of Surgery

Qi-Mei Tang

Third Military Medical University Daping Hospital and Research Institute of Surgery

Min Chen

Third Military Medical University Daping Hospital and Research Institute of Surgery

Qian Huang

Third Military Medical University Daping Hospital and Research Institute of Surgery

Heng-Liu Huang

Third Military Medical University Daping Hospital and Research Institute of Surgery

\section{Liu Wang}

Third Military Medical University Daping Hospital and Research Institute of Surgery

\section{Ning Su}

Third Military Medical University Daping Hospital and Research Institute of Surgery

\section{Xian-Ge Sun}

Third Military Medical University Daping Hospital and Research Institute of Surgery

\section{Wei-Ping Lu}

Third Military Medical University Daping Hospital and Research Institute of Surgery

\section{Shao-Li Deng}

Third Military Medical University Daping Hospital and Research Institute of Surgery

Qing Huang

Third Military Medical University Daping Hospital and Research Institute of Surgery

\section{Research Article}

Keywords: Coronavirus disease 2019, Severe acute respiratory syndrome coronavirus 2, Real-time fluorescent PCR, Methodology evaluation

Posted Date: February 23rd, 2022

DOI: https://doi.org/10.21203/rs.3.rs-1340742/v1

License: () (1) This work is licensed under a Creative Commons Attribution 4.0 International License. 
Page 2/14 


\section{Abstract}

Background: it is necessary to evaluate real-time fluorescent reverse-transcription PCR (RT-PCR) methods to detect the nucleic acids of severe acute respiratory syndrome coronavirus 2 (SARS-CoV-2).

Methods: considering lack of positive specimens in some particular locations in China, the specimens from healthy individuals were used to perform the methodology evaluations, in which the indexes were the differences of quantification cycle values $(\Delta C \mathrm{q})$ between human-derived internal reference control (IRC) genes of a specimen and quality control gene (QC). A series of experiments was conducted to evaluate various factors that might affect the results, such as types of virus transport media, methods of specimen pretreatment and template preparation, specimen vortex strength, specimen storage temperature, and duration.

Results: it was better to store specimens in normal saline (NS) transport medium, release more virus particles from swabs by vortex mixing, extract nucleic acids with centrifugation methods, and perform amplification assays timely. The above-mentioned options and optimum conditions were further confirmed using SARS-CoV-2 pseudoviruses and positive clinical specimens.

Conclusions: this study provides a solution for the accurate detection of SARS-CoV-2. Specifically, this study also indicates that the routine specimens from healthy individuals could be used to methodological evaluation of real-time fluorescent RT-PCR targeting SARS-CoV-2, of which the indexes were the $\Delta C q$ values.

\section{Introduction}

Since its outbreak, the coronavirus disease 2019 (COVID-19) has spread at home and abroad in a short period, threatening human health worldwide (1). On March 11, 2020, the World Health Organization announced that the global COVID-19 spread was a pandemic (2). Up to December 18, 2021, more than 270 million people have been infected worldwide, approximately 5.3 million individuals have died, and the number of infected people continues to rise (3). COVID-19 is a viral disease caused by the severe acute respiratory syndrome coronavirus 2 (SARS-CoV-2) virus. Early diagnosis is essential to identify COVID-19 cases to provide correct treatment in time and effectively prevent this infectious disease $(4,5)$. In China, from the second edition to the latest edition (the seventh) of the Diagnosis and Treatment Program issued by the National Health Committee, real-time fluorescent reverse-transcription PCR (RT-PCR) has been considered one of the golden standards to confirm suspected patients with COVID-19.

Until now, many scientific research institutions and medical enterprises in China conducted studies to develop SARS-CoV-2 nucleic acid detection kits. Many kinds of kits targeting SARS-CoV-2 were commercially available in China. Because there are various methods to preserve the collected oropharyngeal swabs (e.g., different virus transport media), pretreat specimens, and prepare nucleic acid templates, it was essential to perform methodology evaluations to screen the optimum conditions to ensure reliable results of commercial kits targeting SARS-CoV-2. However, there has been no comparison 
reports of the above-mentioned steps. It might be the reason that several RT-PCR detection times were needed to confirm suspected cases of COVID-19 $(6,7)$. Therefore, it is indispensable to evaluate the appropriate methods of SARS-CoV-2 nucleic acid detection to guide laboratory technicians to select the most proper methods to ensure reliable diagnostic results for the clinic.

SARS-CoV-2 positive specimens should be used to perform methodology evaluations $(8,9)$. However, there have been only a few COVID-19 cases outside the Hubei province in China since the COVID-19 pandemic outbreak. Thus, it is not easy to access enough COVID-19 specimens to perform the methodology evaluation for laboratories outside Hubei province. For example, of the approximately 5,000 specimens analyzed in our hospital, only two showed positive results. Therefore, it is speculated that the amplification efficiencies of human-derived internal reference control (IRC) genes could indirectly reflect that of targeted SARS-CoV-2. Given that the respiratory epithelial cells are infected in patients, the nucleic acids of SARS-CoV-2 could be co-extracted along with the human-derived IRC templates $(10,11)$. The amplification of human RNA has been used as an IRC for most commercial kits targeting SARS-CoV-2 $(12,13)$.

In this study, the difference of quantification cycle values $(\Delta C \mathrm{q})$ of human-derived IRC genes between a specimen and a quality control gene (QC) was used as the index to evaluate the specific, commercial available real-time fluorescent RT-PCR kits targeting SARS-CoV-2. The optimum conditions were further confirmed with pseudovirus and positive specimens of SARS-CoV-2.

\section{Materials And Methods}

\section{Collection of various specimens and pseudowires}

All specimens were oropharyngeal swabs (Medico, Guangdong, China), of which the negative and positive specimens came from a healthy volunteer and COVID-19 confirmed cases, respectively. The ethics committee of Daping Hospital approved this study. Written informed consent was obtained from healthy volunteers, patients, or their family members before the specimen collection. Additionally, a pseudovirus carrying an $\mathrm{N}$ gene fragment of SARS-CoV-2 (Zeesan Biotech, Shanghai, China) was also used in this study. After collection, the specimens were immediately detected or stored at $4^{\circ} \mathrm{C}$ or room temperature (RT) for a given duration.

\section{Pretreatment Of The Various Specimens And Pseudovirus}

The three kinds of virus transport media analyzed were normal saline transport medium (NS; Kelly, Sichuan, China), Nanxin transport medium containing guanidine salt (NX; Longs, Guangdong, China), and Young transport medium that did not contain guanidine salt (YK; Yocon, Beijing, China). According to the evaluation experiments requirements described in the following sections, oropharyngeal swabs collected from healthy volunteers and COVID-19 confirmed cases were stored in at least one of the three media. After being vortex-mixed for $30 \mathrm{~s}$, each specimen was further divided into three parts. Finally, one part 
was inactivated in a water bath at $56^{\circ} \mathrm{C}$ for $10 \mathrm{~min}$ (IWS), one of the other two was inactivated in a metal bath at $56^{\circ} \mathrm{C}$ for $10 \mathrm{~min}$ (IBS), and the last one was not inactivated (NI). Some specimens were spiked with a specific concentration of pseudovirus particles.

\section{Preparation Of Nucleic Acid Templates}

After the specimen pretreatment, three types of template preparation methods were performed, i.e., magnetic beads (MB), centrifugation (CF), and the one-step (OS) method. For the MB method, $400 \mu \mathrm{L}$ of the specimen was used to extract nucleic acids according to manufacturer's introductions (Sansure Biotech, Hunan, China), and the templates were eluted with $100 \mu \mathrm{L}$ of sterile water. For the CF method, $400 \mu \mathrm{L}$ of the specimen was centrifuged at $13,000 \mathrm{~g}$ for $10 \mathrm{~min}$, and the pellets were resuspended in 100 $\mu \mathrm{L}$ of nucleic acid release agent (Insure Biotech). For the OS method, $50 \mu \mathrm{L}$ of the specimen was directly gently mixed with $50 \mu \mathrm{L}$ of the nucleic acid releasing agent (Manure Biotech).

\section{Real-time Fluorescent Rt-pcr}

A commercial available SARS-CoV-2 nucleic acid detection kit (Sansure Biotech) was used to analyze various specimens for the ORF1ab and N genes of SARS-CoV-2 and the human-derived IRC genes (i.e., $R$ Nase $\mathrm{P}$ ). The same $\mathrm{QC}$ reactions were included within each batch of experiments to serve as positive or negative controls. All reactions were performed in duplicate to confirm reproducibility. The amplifications were performed on CFX96 PCR System (Bio-Rad; Bio-Rad Lab. Inc., USA) or Cobas Z480 (Roche; Roche Molecular Diagnostics, Pleasanton, $\mathrm{CA}$ ) instruments. The reaction systems and procedures were conducted according to the instructions of the kits.

\section{Statistical analysis}

The $\Delta C$ q values of human-derived IRC genes between the specimen and QC (i.e., $\Delta C q=C q S p e c i m e n-$ $C q Q C$ ) were used as an index to evaluate amplification efficiencies of the currently used commercial RTPCR kits. ANOVA and Tukey's tests were used to analyze the data.

\section{Results}

\section{Analytical performance evaluation of various combinations of specimen pretreatment and template preparation methods}

Ten specimens of oropharyngeal swabs collected from healthy individuals were stored in NS medium. Each specimen was pretreated with three methods (i.e., IWS, IBS, and NI) followed by nucleic acid 
extractions with three methods (i.e., MB, CF, and OS). All reactions were performed in duplicate on both Bio-Rad and Roche instruments.

There were statistically significant differences for $\Delta C$ q values between various template preparation methods $(p<0.05)$ on both Bio-Rad and Roche instruments (Fig. 1). Moreover, there were no differences between template preparation methods and specimen pretreatment ( $p>0.05$; data not shown).

Further analysis results of various template preparation methods showed that for both Bio-Rad and Roche instruments, there were statistically significant differences between the $\Delta C$ q values of CF methods and those of both MB and OS methods $(p<0.01)$. No differences were identified between the $\Delta C q$ values of MB and OS methods $(p>0.05)$. The average $\triangle C q$ value of CF methods was lower than that of MB and OS methods for both instruments, i.e., 3.209 and 2.576 for the Bio-Rad instrument, respectively, and 2.760 and 3.558 for the Roche instrument, respectively. These results indicated that the CF methods had the best analytical performance under the various conditions tested.

\section{Analytical performance evaluation of various transport media and specimen vortexing strength}

Nine specimens of oropharyngeal swabs were collected from three healthy individuals. The specimens of each person were stored in NS, YK, and NX virus transport media. For each specimen, various methods of specimen pretreatment and template preparation were performed as mentioned in the previous sections. All reactions were performed in duplicate on both Bio-Rad and Roche instruments. For various kinds of virus transport media (i.e., NS, YK, and NX), the analytical performance of both specimen pretreatment (i.e., IWS, IBS, and $\mathrm{NI}$ ) and template preparation (i.e., $\mathrm{MB}, \mathrm{CF}$, and $\mathrm{NI}$ ) methods were similar to those of the previous sections. Moreover, there were no interactions between methods of virus transport medium, template preparation, and specimen pretreatment $(p>0.05)$.

Further analysis results showed that the transport medium had significant effects on $\Delta C q$ values for both Bio-Rad and Roche instruments $(p<0.01)$. For NS, YK, and NX media, the average $\Delta C q$ values of Bio-Rad instruments were $0.801,1.936$, and 3.733 , respectively, and that of the Roche instruments were 0.777 , 1.043 , and 2.901, respectively. Statistical analysis results indicated that there were significant differences between $\Delta C q$ values of various kinds of transport media $(p<0.05)$ for both instruments, except for that there were no differences $(p=0.865)$ between those of NS and YK media for the Roche instrument (Fig. 2A). The NS and NX media had the best and worst analytical performance, respectively (for both instruments). Notably, the amplification failed when transport medium was NX and the template preparation method was OS. In the experiment to evaluate the vortex strength's analytical performance, the results showed that the average $\Delta C$ q value of vigorous mixing was 0.296 smaller than that of gentle mixing. However, there was no statistical difference ( $p=0.601$; Fig. $2 \mathrm{~B})$.

\section{Analytical performance evaluation of storage temperature and duration}


Six specimens of oropharyngeal swabs were collected from three healthy individuals. Two specimens from each person were stored in NS and YK virus transport media. Then, each specimen was evenly divided into three parts, of which the first part was tested immediately (i.e., 0 h), and the second and third parts were stored at $4^{\circ} \mathrm{C}$ and under RT conditions, respectively, followed by a test at $12 \mathrm{~h}$ and $24 \mathrm{~h}$, respectively. Nucleic acid extractions were conducted using NI specimen pretreatment followed by CF template preparation. All reactions were performed in duplicate on only the Bio-Rad instrument.

For both NS and YK mediums, it was found that the $\Delta C$ q values were increased with the extension of duration at $4^{\circ} \mathrm{C}$ or RT storage temperature. At 12 or $24 \mathrm{~h}$ storage duration, the $\Delta C q$ values between specimens stored at $4^{\circ} \mathrm{C}$ and RT were close when NS media were used (Fig. 3A) and significantly different when YK mediums were used (Fig. 3B). These results indicated that the storage temperature had little effect on NS medium analytical performance and significant effects on that of the YK medium. Based on the comparative analysis of the $\Delta C$ q values at various storage conditions, we conclude that it is better to use NS medium in clinical practice, and to store the specimens at $4^{\circ} \mathrm{C}$ when the YK medium is used.

\section{Clinical evaluation of analytical parameters using pseudovirus and COVID-19 specimens}

Two specimens of oropharyngeal swabs from healthy individuals were collected and stored in the NS transport medium. Each specimen was then evenly divided into two parts. Definite concentrations of pseudovirus carrying fragments of the SARS-CoV-2 Ngene were spiked into pure NS media, and one part of the abovementioned NS media containing specimens from healthy individuals. Finally, three types of specimens carrying various templates, i.e., human IRC and SARS-CoV-2 $N$ genes (Fig. 4A), purely human IRC genes (Fig. 4B), and SARS-CoV-2 Ngenes (Fig. 4C), were directly used to extract nucleic acids with three specific methods, i.e., CF, MB and OS methods. All reactions were performed in duplicate and using only the Bio-Rad instrument.

The results of all specimens showed close $C q$ values for the IRC or $N$ genes between the MB and OS methods (Fig. $4 A-C$ ). The $C$ q values of the IRC genes using the $C F$ methods were smaller than that of both the MB and OS methods (Fig. 4A and 4B), which followed previous sections, i.e., CF methods had better analytical performance (Figs. 1 and 2). However, the $C$ q values of the $N$ genes of $C F$ methods were more extensive than those of both the MB and OS methods (Fig. 4A and 4C), which were inconsistent with those of the IRC genes. Moreover, similar results were found for the $\mathrm{Ngene}$, even increasing the centrifuge force and time (data not shown). This might be because the pseudovirus could not be concentrated with human cells together using the CF methods.

Finally, two specimens of oropharyngeal swabs from COVID-19 confirmed cases were collected and stored in NS transport media. They were directly used to extract nucleic acids with three specific methods, i.e., the CF, MB, and OS methods. The results of all specimens showed close $C q$ values of the IRC or $N$ genes between the MB and OS methods (Fig. 4D). However, the $C q$ values of the IRC and $N$ genes of CF methods were smaller than those of the MB and OS methods (Fig. 4D), consistent with analytical 
performance using human IRC as an index in previous sections. Moreover, it was notable that there was visible amplification curves of SARS-CoV-2 ORF1ab genes only in reactions using the CF method (Fig. 4D). These results further confirmed that the CF method was better than both MB and OS methods. In addition, they strongly suggest that the amplification efficiencies of IRC genes can evaluate the analytical performance of real-time fluorescent RT-PCR kits targeting SARS-CoV-2.

\section{Discussion}

Under the current epidemic conditions, as a golden standard to diagnose COVID-19 suspected cases, realtime fluorescent RT-PCR targeting SARS-CoV-2 is routinely implemented in most large- and medium-sized laboratories in China (14-16). Several types of specimens can be used to detect SARS-CoV-2 nucleic acids, such as respiratory nasal swabs, oropharyngeal swabs, sputum, and others (17-19). However, many factors might affect SARS-CoV-2 nucleic acid detection's analytical performance, such as types of virus transport media, methods of specimen pretreatment and template preparation, specimen storage temperature, and duration. Conventionally, it is better to use SARS-CoV-2 positive specimens to evaluate the performance of real-time fluorescent RT-PCR kits $(8,9)$. However, considering the biosafety and lack of positive specimens in most laboratories outside the Hubei province in China, it is critical to find alternative methods to evaluate SARS-CoV-2 nucleic acid detection's analytical performance.

As the virus infects human respiratory epithelial cells, SARS-CoV-2 particles could be co-extracted with human cells containing the IRC genes that could be detected by most commercially available real-time fluorescent RT-PCR kits $(10,11)$. Therefore, the abundance of IRC genes could be theoretically used to represent the extraction or preparation efficiency of SARS-CoV-2 nucleic acids. Compared with conventional strategies using specimens from COVID-19 confirmed cases, the current evaluation methods using specimens from healthy individuals are much more straightforward, safer, and more efficient to perform evaluations, namely in routine clinical laboratories that cannot implement demanding biosafety measures. Based on the above considerations, the specimens of oropharyngeal swabs collected from healthy individuals were used to evaluate the analytical performance of SARS-CoV-2 nucleic acid detection, in which the indexes were the $\triangle C$ q values between IRC genes of a specimen and QC. Serial experiments were conducted to evaluate various factors that might affect analytical performance, such as methods of specimen pretreatment and template preparation, types of virus transport medium, storage temperature, and duration. Being extremely valuable in theoretical and practical significance, the aforementioned $\Delta C$ q values' analytical performances were confirmed by pseudovirus and COVID-19 specimens.

Compared with both MB and OS methods of template preparations, the CF methods showed the best analytical performance. Using a more extensive collection of initial volumes of specimens, the CF methods exhibited theoretical advantages and practical results compared to OS methods (Fig. 1-4). Although using the same initial volumes of specimens, the CF methods also showed better performance than the MB methods (Fig. 1-4), which might be associated with the relatively lower recovery rate of MB methods (19). Notably, in our clinical practice, the CF methods also exhibited reliable results for one 
another commercial available real-time fluorescent RT-PCR kit (Daan Gene, Guangdong, China; data not shown).

In the experiments using templates containing pseudovirus fragments, the $C$ q values of IRC or $N$ genes were close, which further confirmed that the MB methods had low extraction efficiencies because larger initial volumes of specimens were used in MB methods. However, compared with MB and OS methods, it was interesting to observe that the CF methods exhibited lower amplification efficiency for $\mathrm{Ngenes}$ (Fig. 4A and 4C), which was inconsistent with the data for the IRC genes (Fig. 1-3 and 4B). The reasons for these results might be that the pseudovirus particle could not be deposited well in CF methods, which would result in a low amplification. However, the above problems do not exist in clinical specimens. The reason might be that the SARS-CoV-2 mainly existed in the epithelial cells or adhered to the cell surface. Therefore, the SARS-CoV-2 particles could be co-deposited with the epithelial cells when CF methods were used $(10,11)$. The abovementioned speculation was confirmed by subsequent experiments using clinical COVID-19 specimens, in which both IRC and $N$ genes exhibited better amplification than the CF method, and the amplification of ORF1ab genes was exhibited only in CF methods (Fig. 4D). The previous results suggested that methodology evaluations based on human IRC genes could be implemented as acceptable strategies to explore the analytical performance of SARS-CoV-2 nucleic acid detection.

A standard limitation of the PCR-based method is failed amplification due to the presence of PCRinhibitory substances in the specimens, such as the heme compounds found in blood, aqueous and vitreous humor, heparin, urine, or polyamines, among other compounds (20). Based on the current comparative analysis on various types of virus transport media, it was essential to select the kinds of virus transport media when the CF or OS method of template preparation is planned to be used in realtime fluorescent RT-PCR targeting specific pathogens. For example, due to the presence of an amplification inhibitor, i.e., guanidine salt $(21,22)$, the analytical performance of the NX medium was always lower than that of both NS and YK media (Fig. 2A). Considering the biosafety of RT-PCR detection targeting SARS-CoV-2, it is better to inactivate pathogens using water or a metal bath before any procedures because there are no significant differences between the various specimens pretreatment (Fig. 1). For the other factors that might affect SARS-CoV-2 nucleic acid detection's analytical performance, it is better to implement the analysis in time (Fig. 3) and release SARS-CoV-2 particles from oropharyngeal swabs by vortex mixing (Fig. 2B).

\section{Conclusion}

Based on the comparison of the $\triangle C$ q values of IRC genes between specimens and QC, routine specimens from healthy individuals can be used to evaluate the methodological evaluation of real-time fluorescent RT-PCR targeting SARS-CoV-2. Among various factors that might affect performance mentioned before, it is recommended to store specimens in NS medium, inactivate pathogens using water or a metal bath, release more virus particles from swabs by vortex mixing, extract nucleic acids using CF methods, and perform amplification assays in a timely manner. Being extremely valuable in theoretical and practical 
significance, the abovementioned findings were further confirmed by testing pseudovirus and clinical positive SARS-CoV-2 specimens.

\section{Declarations}

Conflicts of interest The authors report no conflict of interest.

Author contributions SLD and QH conceived and designed the study. XDR, QMT, MC, QH and HLH performed the experiments and interpreted the results. XDR and QH wrote the manuscript. LW and NS edited the figures and references in the manuscript. XGS and WPL assisted in conducting the experiments and analyzed the data. All authors read and approved the final version of the manuscript.

Ethics approval All procedures performed in studies involving human participants were in accordance with the ethical standards of the institutional and national research committee and with the $1964 \mathrm{Helsinki}$ declaration and its later amendments or comparable ethical standards.

Consent to participate Informed consent was obtained from all individual participants included in the study.

Consent to publish The authors affirm that human research participants provided informed consent for publication

Funding This study was partly supported by the 2019-nCoV Emergency Research Project (Grant No. 313 CWS20C008), the Military Logistics Scientific Research Project (Grant No. 2019HQZX06), the Military Medical Frontier Innovation Ability Training Program (Grant No. 2019CXJSB005), and the University Outstanding Talent Support Program.

\section{References}

1. Lake MA. What we know so far: COVID-19 current clinical knowledge and research. Clin Med. 2020. doi: 10.7861/clinmed. 2019-coron.

2. World Health Organization: Coronavirus disease (COVID-19) outbreak situation [Internet]. [cited 2020 May 7]. Available from: https://www.who.int/dg/speeches/detail/who-director-general-s-openingremarks-at-the-media-briefing-on-covid-19-11-march-2020

3. World Health Organization: Coronavirus disease (COVID-19) outbreak situation. [updated 2020 September 21, cited 2020 July 21]. Available from: https://covid19.who.int/

4. Phuong NH, Kwak C, Heo CK, et al. Development and characterization of monoclonal antibodies against nucleoprotein for diagnosis of influenza a virus. J Microbiol Biotechn. 2018; 28: 809-15.

5. Sun G, Vinh NQ, Matsuoka A, et al. Design an easy-to-use infection screening system for non-contact monitoring of vital-signs to prevent the spread of pandemic diseases. Conf Proc IEEE Eng Med Biol 
Soc. $2014 ; 2014: 4811-4$.

6. Xie X, Zhong Z, Zhao W, et al. Chest CT for typical 2019-nCoV pneumonia: relationship to negative RT-PCR testing. Radiology. February 12; 2020 as doi:10.1148/radiol.2020200343.

7. Ruan ZR, Gong P, Han W, Huang MQ, Han M. A case of 2019 novel coronavirus infected pneumonia with twice negative 2019-nCoV nucleic acid testing within 8 days. Chin Med J (Engl). 2020. doi:

10.1097/CM9.0000000000000788

8. Carrillo-Ávila JA, de la Puente R, Catalina P, et al. Evaluation of RNA purification methods by using different blood stabilization tubes: identification of key features for epidemiological studies. BMC Res Notes. 2020; 13: 77.

9. Kodama M, Okimoto T, Mizukami K, et al. Evaluation of a novel anti-H. pylori antibody detection kit by latex turbidimetric immunoassay. Clin Lab. 2019; 65

10. Chu DKW, Pan Y, Cheng SMS, et al. Molecular diagnosis of a novel coronavirus (2019-nCoV) causing an outbreak of pneumonia. Clin Chem. 2020; 66: 549-55.

11. Chan JF, Yip CC, To KK, et al. Improved molecular diagnosis of COVID-19 by the novel, highly sensitive and specific COVID-19-RdRp/Hel real-time reverse transcription-polymerase chain reaction assay validated in vitro and with clinical specimens. J Clin Microbiol. 2020. doi:

10.1128/JCM.00310-20.

12. Xu H, Zhong L, Deng J, et al. High expression of ACE2 receptor of 2019-nCoV on the epithelial cells of oral mucosa. Int J Oral Sci. 2020; 12: 8.

13. Zhu N, Zhang D, Wang W, et al. A Novel Coronavirus from Patients with Pneumonia in China, 2019. N Engl J Med. 2020; 382: 727-33.

14. Huang X, Wei F, Hu L, et al. Epidemiology and clinical characteristics of COVID-19. Arch Iran Med. 2020; 23: 268-71.

15. Tian S, Hu N, Lou J, et al. Characteristics of COVID-19 infection in Beijing. J Infect. 2020; 80: 401406.

16. Chen Y, Chen L, Deng Q, et al. The presence of SARS-CoV-2 RNA in the feces of COVID-19 patients. J Med Virol. 2020. doi: 10.1002/jmv.25825.

17. Kannan S, Shaik Syed Ali P, Sheeza A, et al. COVID-19 (Novel Coronavirus 2019) - recent trends. Eur Rev Med Pharmacol Sci. 2020; 24: 2006-11.

18. Wang W, Xu Y, Gao R, et al. Detection of SARS-CoV-2 in Different Types of Clinical Specimens. JAMA. 2020. doi: 10.1001/jama.2020.3786.

19. Peng L, Liu J, Xu W, et al. SARS-CoV-2 can be detected in urine, blood, anal swabs and oropharyngeal swabs specimens. J Med Virol. 2020. doi: 10.1002/jmv.25936.

20. Akbariqomi M, Heidari R, Gargari SS, et al. Evaluation and statistical optimization of a method for methylated cell-free fetal DNA extraction from maternal plasma. J Assist Reprod Genet. 2019; 36: 1029-38. 
21. Fredricks DN, Relman DA . Improved amplification of microbial DNA from blood cultures by removal of the PCR inhibitor sodium polyanetholesulfonate. J Clin Microbiol. 1998; 36: 2810-6.

22. Root BE, Agarwal AK, Kelso DM, et al. Purification of HIV RNA from serum using a polymer capture matrix in a microfluidic device. Anal Chem. 2011; 83: 982-8.

23. Nakamichi K, Lim CK, Saijo M. Stability of JC virus DNA in cerebrospinal fluid specimens preserved with guanidine lysis buffer for quantitative PCR testing. Jpn J Infect Dis. 2014; 67: 307-10.

\section{Figures}
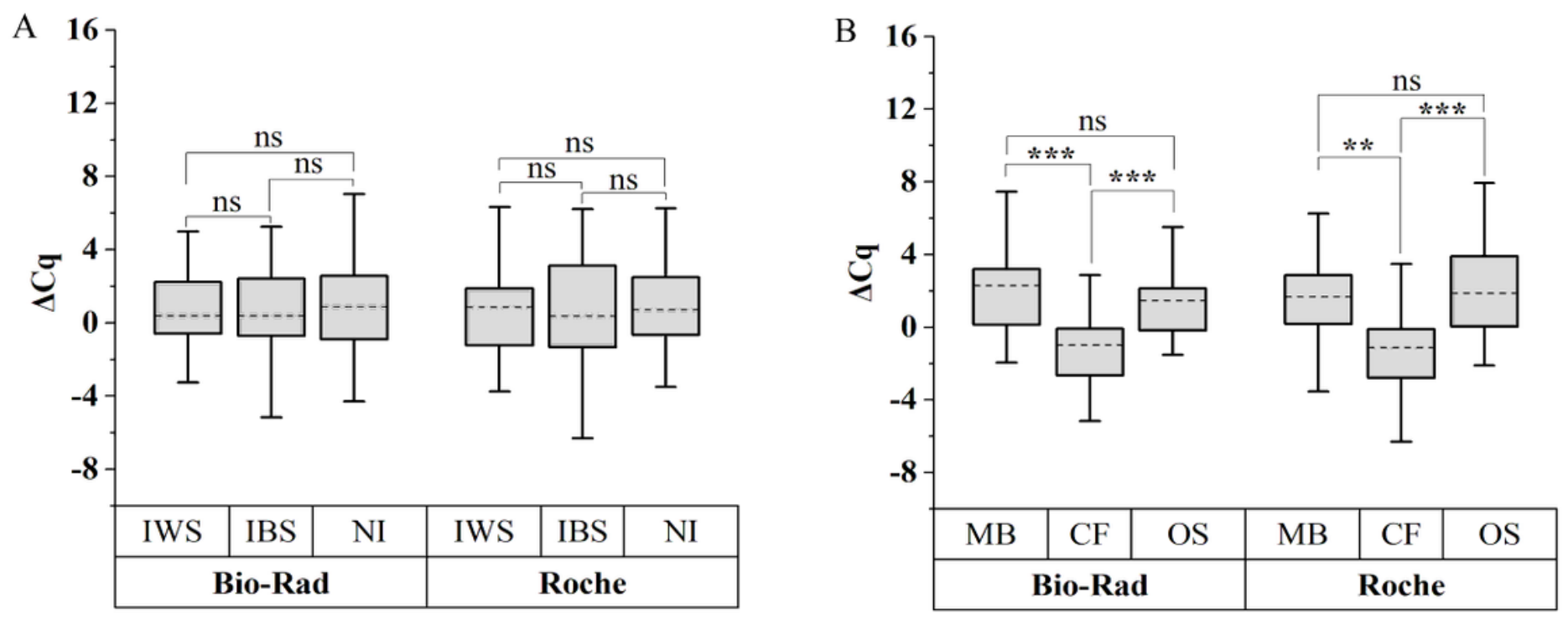

Figure 1

Features of various combined performances between specimen pretreatment and template preparation methods

The IRC $\Delta C$ q values in box chart plots $(n=30)$ of various specimen pretreatments (panel $A$ ) or template preparations (panel B) methods. Parallel amplifications were performed on Bio-Rad and Roche instruments. Asterisks indicate that the $p$-value was a significant difference (i.e. $*$ : $p<0.05$; $* \star$ : $p<0.01$; $* \star *$ : $p<0.001)$. The ns indicates that there is no statistically significant difference. 

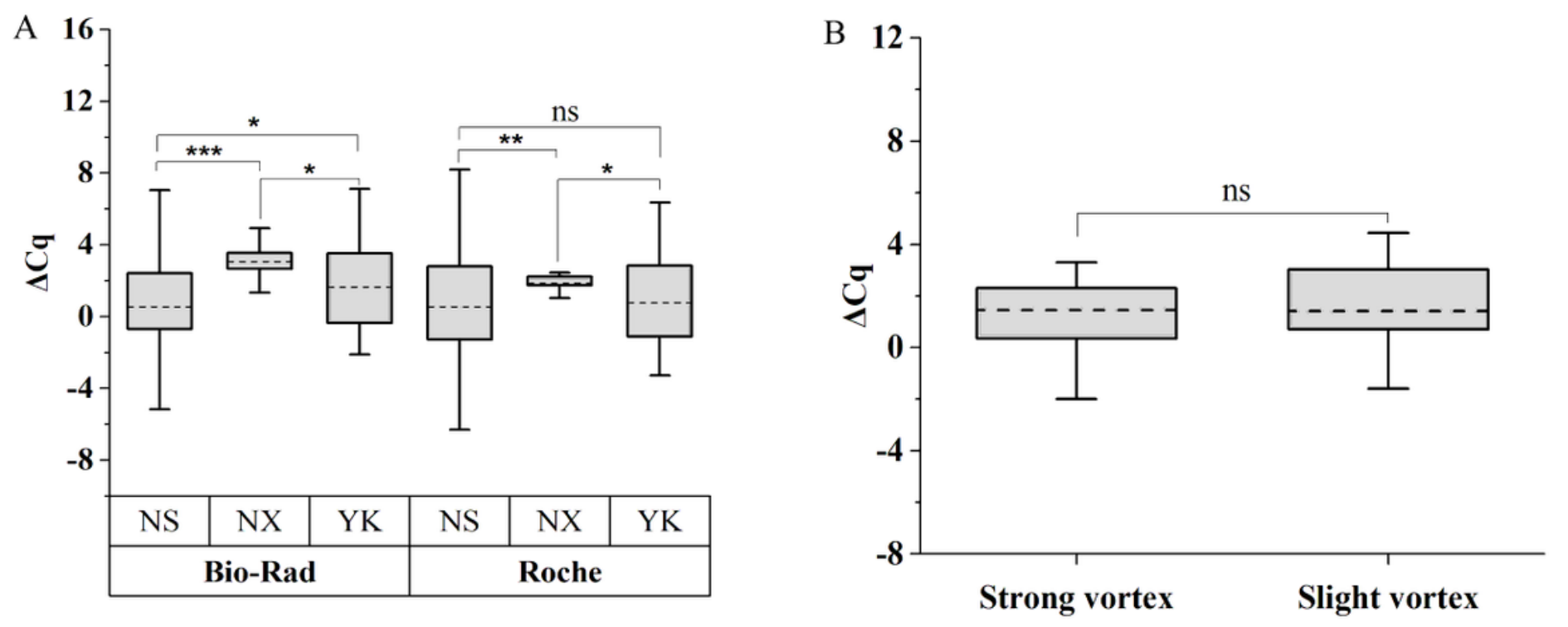

Figure 2

Features of various transport media and specimen vortex strength.

The IRC $\Delta$ Cq values in box chart plots of various transport mediums (panel $A ; n=9$ ) or vortex strengths (panel $B ; n=6$ ). Parallel amplifications were performed on Bio-Rad and Roche instruments. Asterisks indicate that the $p$-value reports a significant difference (i.e. *: $p<0.05 ; * *: p<0.01 ; * \star *: p<0.001$ ). The ns indicates that there is no statistically significant difference.
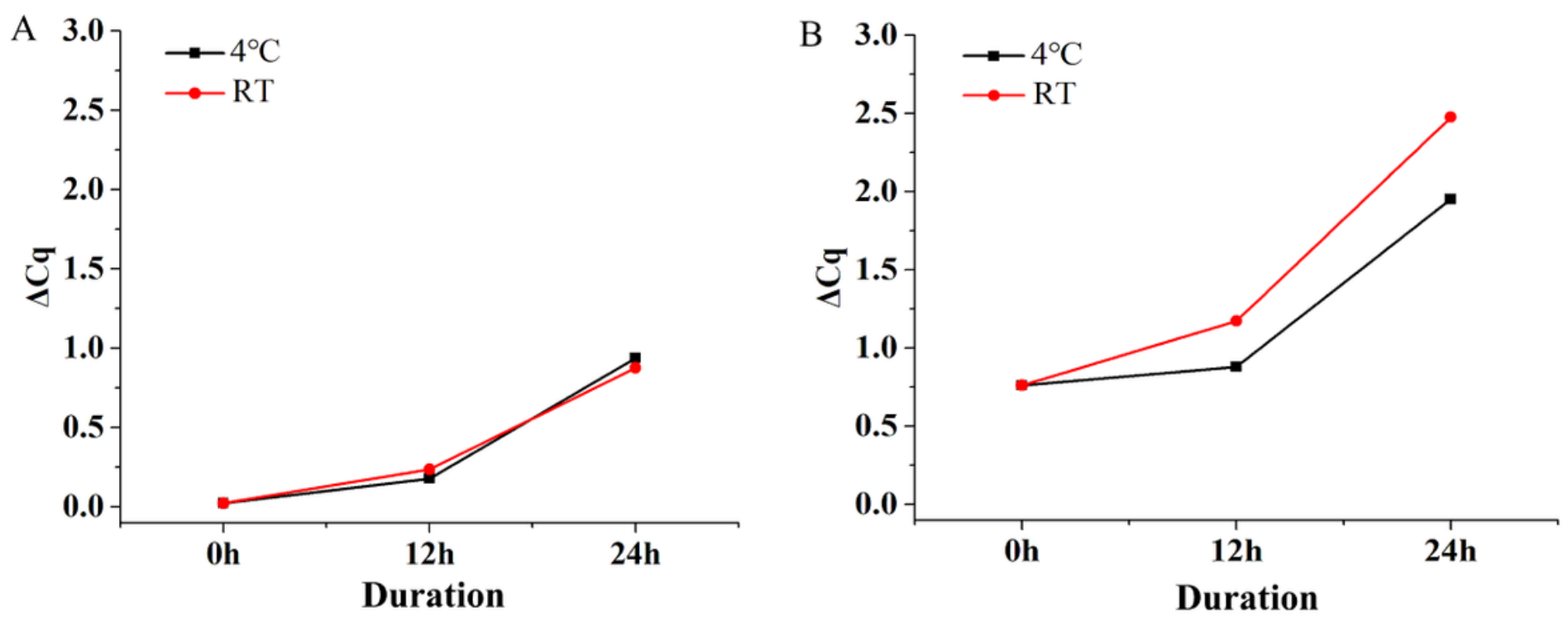

Figure 3

Features of various storage temperature and duration conditions.

The average $\Delta C q$ values $(n=3)$ under conditions of various storage temperatures and durations 
when NS (panel A) or YK (panel B) media were used.
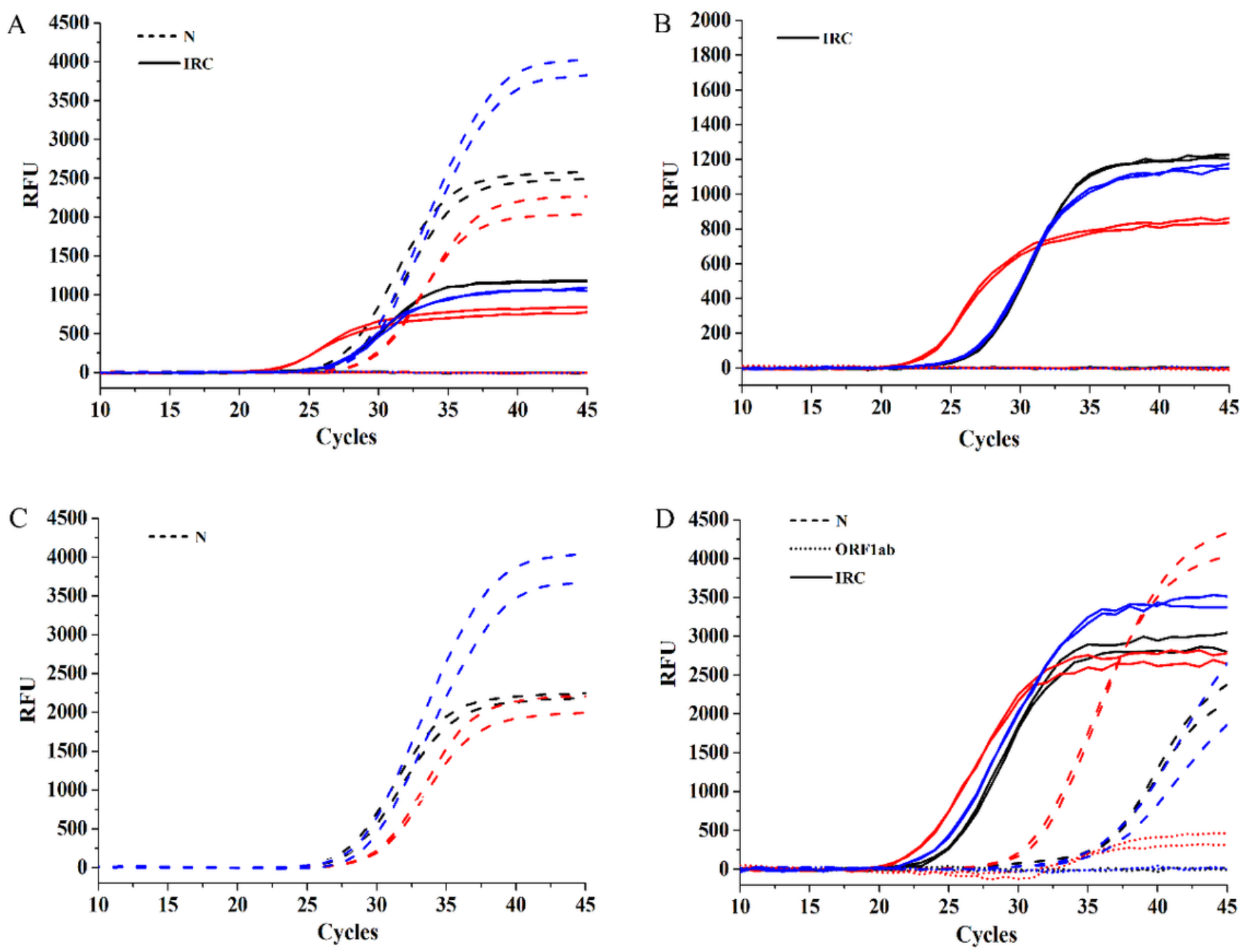

Figure 4

Example results of pseudovirus and SARS-CoV-2 patient specimens.

Results of a mixture containing various templates, i.e., pseudovirus $\mathrm{N}$ and human IRC genes (panel $\mathrm{A}$ ), human IRC genes (panel $\mathrm{B}$ ), the pseudovirus $\mathrm{N}$ genes (panel $\mathrm{C}$ ), and the positive specimens (panel $\mathrm{D}$ ). The template preparation methods are indicated with various colors, i.e., the red, black, and blue lines indicate the $\mathrm{CF}, \mathrm{MB}$, and $\mathrm{OS}$ methods, respectively. 